\section{Labelling of Drugs}

SIR,-The report of the Committee on Safety of Drugs for 1965 expressed the view that it would conduce greatly to the safe use of drugs if containers of prescribed medicines were labelled with the name of the medicine unless otherwise specified by the doctor. This stimulated a considerable degree of comment in the lay press.

The Committee is not alone in advocating this view. Nevertheless, many of your readers may be unaware of how widely it is already shared, and they may be interested, therefore, in seeing the text of a letter I sent earlier this year to the Presidents of the Royal Colleges in England, Edinburgh, and Glasgow, to the Presidents of the College of Medical Practitioners and the Royal Medico-Psychological Association, and to the Deans of the Faculties of Dental Surgery and Anaesthetics of the Royal College of Surgeons, England:

"The Committee on Safety of Drugs are very conscious that the adequacy of toxicity testing, clinical trials, and the monitoring of adverse reactions are not the only factors in the safe use of drugs. Patients, the general public, and particularly prescribing doctors in practice and in hospital have an important role to play. The Committee have always believed that it would conduce greatly to the safe use of drugs if their containers were labelled with the name of the medicine ordered unless otherwise specified by the prescriber.

"We are convinced that the great majority of the medical profession strongly favour this reform, yet the British Medical Association and the Pharmaccutical Society have replied to our repeated representations that in their view the time is not yet ripe for the change, though the have agreed to accept the convention N.P. (nomen proprium) written on the prescription to indicate that the prescriber wishes the name of the drug to appear on the label.

"We believe that a positive rather than a negative approach should be adopted and that the standard practice should be that all containers of medicine should be labelled with the name of the drug unless otherwise specified. No doctor can practise good medicine withou being thoroughly conversant with the nature of the drugs his patient may be taking. Yet as the following examples show he may, with the present practice, be denied this information when he needs it:

"(1) A patient often needs his prescription to be renewed when his own doctor is not available and his partner is doing duty for him Records may be inaccessible or incomplete. In such cases the patient's treatment could be interrupted or pre udiced by lack of knowledge, which might be avoided if the container of the medicine he had been taking was properly labelled. Whether we like it or not it is becoming more common for a patient to be looked after by more than one doctor and if the Doctor's Charter, as envisaged by the British Medical Association, becomes operative, this will become still more common.

“(2) In outpatient departments, the details of the patient's previous treatment are not infrequently omitted from the practitioner's accompanying letter and at present the consultant receives no information from the box of tablets which is often produced.

" (3) Conversely, it is frequently the practice for patients on discharge from hospital to be given a few days' supply of medicine for them to take until more can be prescribed by their own doctors. The consultant's letter, however, is not always dispatched immediately on the patient's discharge and the practitioner remains in ignorance of what medicine his patient is taking, or what he should prescribe.

"(4) Because of the undesirability of anaesthetizing patients taking certain drugs or of operating on them without adequate preparation-for example, corticosteroids-it is essential for the anaesthetist and surgeon to know what medicines the patient may have been taking previously. In emergencies it is not always possible to get in touch with the doctor of the patient, and the tablets found in his possession may give a valuable clue if properly labelled.

" (5) The likelihood of sensitivity reactions or of dangerous interactions between one drug and another (for example, mono-amine oxidase inhibitors and ephedrine) is lessened by adequate labelling.

(6) There are over 5.000 cases annually of accidental poisoning in this country, with a high proportion among children, approximately half of which are due to drugs-mainly tablets and capsules. To be effective, treatment must be prompt and may be handicapped by ignorance of the actual drug taken. The container-sometimes empty-is often brought with the patien but when it is only labelled 'The Tablets' the information is unhelpful.

"The suggestion that patients should usually be kept in ignorance of their treatment is quite inconsistent with contemporary medical thought. We realize of course that there are occasions when, in the patient's interests, it may be undesirable for him to know what drug he is being given. This is particularly the case when placebo or 'terminal drugs' are prescribed. Such contingencies, however, are covered by the gualification 'unless otherwise specified by the prescriber.'

"The Minister of Health has told us that this is a cuestion which must be settled by the professions conccrned. We therefore have to demonstrate that informed opinion in the medical profession supports the rrocedure we suggest. It has already been endorsed by the Central Consultants and Specialists Committee, and has been adopted in a considerable number of hospitals. It has become the standard practice in Scandinavian countries and New Zealand without causing any problems. Further progress here depends on the production of evidence of the strength of opinion in the medical profession, to which we would earnestly ask you to lend the weight of your authoritative supnort. It wnuld be particularly valuable if you would publish a statement of your attitude or send me a reply authorizing the Committee to include your support in a public statement of this kind."

The response to this letter was unanimously-even enthusiastically_favourable, and I believe that publication of this fact in your columns would greatly help to resolve any doubts which still remain about the wisdom of changing the present convention of dispensing practice.-I am, etc.,

$$
\begin{gathered}
\text { DERdon S.W.1. } \\
\text { Chairman, } \\
\text { Committee on Safety of Drugs. }
\end{gathered}
$$

\section{Dangers of Immunosuppressive Drugs in Ulcerative Colitis}

SIR,-I am in general agreement with the comments of Dr. F. Avery Jones and his colleagues in their recent letter (4 June, p. 1418).

Some of their points are worth further comment. Whilst I have had no experience in the use of azathiaprine in ulcerative colitis, I believe the same principles govern the use of this drug as in the case of 6-mercaptopurine antimetabolites. I consider it important that the initial dose be the maximum tolerated dose, and that reduction or cessation of therapy depends upon whether success or failure follows this initial trial. It is my conviction that to increase dosage after failure of inadequate therapy is extremely hazardous. I have been using large intermittent doses of 6-mercaptopurine over the last 18 months. These are frequently given in conditions in which there is extensive marrow involvement -for example, acute myeloid leukaemia, carcinomatosis; neutropenia is rarely severe, although total doses of 6-mercaptopurine in excess of $20 \mathrm{~g}$. over six to twelve months are not uncommon. Using the continuous dosage regimen described in my paper severe neutropenia occurred only in Case 6. In this patient a high dosage was necessary to control haemolysis, and the position was complicated by the previous administration of phthalylsulphathiazole for at least four weeks. As a principle great care should be used in the use of antimetabolites when the patient is under current or recent therapy with a drug recognized to be a marrow-depressant, or in the presence of a significant degree of neutropenia.

I must also disagree with Dr. Jones and his colleagues upon the use of the title "immunosuppressive." This title has aetiological connotations which I consider far from proved and potentially misleading. Failure to demonstrate an antigen does not mean that an antibody has arisen de novo. I have given some reasons why I do not consider antimetabolites to be "immunosuppressive" in colitis in my paper, and would like to add to these.

We have been using the dinitrochlorobenzene skin test as described by Aisenberg as an index of the ability of patients to form antibodies. ${ }^{1}$ In six out of the seven patients described in my paper (30 April, p. 1081) we find that, in spite of prolonged therapy with antimetabolites, the response is increased rather than diminished. Thus the response is poor only in one patient (Case 5), and it may be significant that this patient has not attained the symptom-free condition of the other members of the group, and furthermore has a family history of bowel cancer. In general the response to D.N.B. is less active in other patients, who have presented with malignancy superimposed upon colitis.

Antimetabolites remain a crude method of combating ulcerative colitis. Methods do not remain static. I consider that my current regimen of therapy offers some advantages over that described in the paper, and this is briefly summarized. Initially $0.5 \mathrm{~g}$. to $1 \mathrm{~g}$. of 6-mercaptopurine and $100 \mathrm{mg}$. of hydrocortisone are administered, depending upon the physical condition of the patient and acuteness of the condition. The response is clinically evaluated over the next two to three days and therapy repeated every three to seven days. It is unusual to need more than the two to three large doses of 6-mercaptopurine. As soon as definite improvement occurs the dose is reduced to $0.25 \mathrm{~g}$. of $6-$ mercaptopurine with $40 \mathrm{mg}$. of hydrocortisone every two to three days, and when the improvement is stabilized $100 \mathrm{mg}$. of busulphan is given over four days. The 6-mercaptopurine is ceased 14 days after the course of busulphan is completed. Long remissions are occurring with this regimen.-I am, etc.,

Repatriation General Hospital, R. H. D. BEAN.

Heidelberg, Victoria,

REFERENCB

1 Aisenberg, A. C., f. clin. Invest., 1962, 41, 1964. 